\title{
EL LENTO PROGRESO DE LA CIENCIA EN EL TERCER MUNDO*
}

ABDUS SALAM

El premio Nobel de Física de 1979, el paquistaní Abdus Salam, ilustra las dificultades encontradas por las ciencias en el tercer mundo -algo que por lo general se origina en los procesos de toma de decisiones en los países industrializados.

¿Puede transferirse tecnología a los países poco desarrollados sin transferir también ciencia? La respuesta es no. El resultado de tal práctica es desastroso, sin embargo es muy común. ¿Cómo podemos evitar esta dificultad? Creando, dice el Dr. Salam, una Fundación Internacional para el avance del crecimiento científico del Sur dentro del ámbito global. Y también aumentando los fondos para el entrenamiento de científicos de países subdesarrollados. "Después de todo", enfatiza el ganador del Nobel, "la cantidad gastada en un submarino nuclear sería suficiente para financiar 500 centros de investigación por todo un año" ...

Desde su fundación en 1964, el International Center for Theoretical Physics (Centro Internacional de Física Teórica) en Trieste, Italia, ha recibido 22,000 visitas de investigadores en física teórica y experimental; 11,000 de estas visitas

Traducido de: COOPERAZIONE. Italian Ministry of Foreing Affairs. New Series/10 th year, 1985. Supp. to No. 51: 47-51. Traducción de José Ramón Albaine Pons, Fo No. 8. 
fueron realizadas por trabajadores de universidades y centros de investigación científica de naciones en desarrollo.

Con esta experiencia como base, adquirida en los últimos 20 años, la tesis que deseo presentar es la siguiente: con la excepción de algunos países -Argentina, Brasil, China e India- el Tercer Mundo, a pesar de la aceptación reciente de que la ciencia y la tecnología son los sostenedores y su mayor esperanza de mejoramiento económico, han tomado a la ciencia -en contraste con la tecnología- como una actividad marginal. Esto también es verdad para las agencias internacionales de donaciones de los países ricos, de las agencias de las Naciones Unidas y también, y desafortunadamente, de las comunidades científicas de los países desarrollados los que están llamados a ser por su naturaleza los principales aliados de los científicos del Tercer Mundo.

Mi propósito en este artículo es reflexionar sobre la posición de las ciencias en los países en desarrollo y llamar la atención, en particular, al papel de las Naciones Unidas y otras agencias, en el mejoramiento de la situación.

¿Por qué enfatizo que la ciencia en los países en desarrollo ha sido tratada como una actividad marginal? Por dos razones:

Primero: Los hacedores de polítieas, las comisiones prestigiosas (como la Brandt Commission), así como los donantes, hablan uniformemente de los problemas de transferencias de tecnologías hacia los países subdesarrollados como si ese fuese todo el paquete. Es difícil de creer, pero es cierto, que la palabra "ciencia" no figura en el reporte de la Comisión Brandt. Muy pocos en los países subdesarrollados parecen enfatizar que para una efectividad a largo plazo, las transferencias tecnológicas deben siempre ir acompañadas de transferencias científicas; que la ciencia de hoy es la tecnología de mañana y que cuando hablamos de ciencia, ésta debe verse en una amplia base, para que pueda ser efectiva para aplicaciones.

La base científica de todos los productos y procesos se vuelve cada vez más sólida. A mayor ciencia que un nuevo producto o proceso presenta, mayor seguridad de que será competitivo.

La mayoría de los países del Tercer Mundo apenas tienen algo de ciencia creativa. El $90 \%$ del potencial investigativo del mundo está concentrado en 35 países que presentan el $25 \%$ de la población mundial. De ahí la necesidad imperativa para un país del Tercer Mundo de encontrar la estrategia para producir rápidamente una macrotransferencia científica sobre la cual basar su desarrollo. Sin tal transferencia científica un país del Tercer Mundo continuará siendo tecnológicamente, y por lo tanto económica y políticamente, dependiente unilateralmente, dicho de forma más simple, explotado en sus intercambios internacionales.

Yo aún iría más lejos y diría: si uno fuera maquiavélico, se discernirían motivos siniestros entre aquellos que tratan de vendernos la idea de la trans- 
ferencia tecnológica sin la transferencia de la ciencia. No hay nada que haya causado más daño al Tercer Mundo que el slogan de los países ricos de "ciencia relevante". Tristemente este estribillo ha sido repetido en nuestros paises sin pensar, para justificar la parálisis en el vencimiento de toda ciencia.

Segundo: la transferencia científica es efectuada por y para comunidades de científicos. Tales comunidades (en países en desarrollo) necesitan crecer hasta un tamaño crítico tanto en recursos humanos como en infraestructura.

Este crecimiento necesita de políticas científicas sabias, con compromisos a largo plazo, un patronazgo generoso, autogobierno y contactos internacionales libres. Más aún, en nuestros países, el científico de alto nivel debe ser requerido para jugar un papel en la construcción de la nación en igualdad de condiciones con los planificadores, economistas y técnicos. Pocos países subdesarrollados han promulgado tales políticas, pocas agencias de ayuda han tomado como su rol el impulsar y ayudar en la construcción de la infraestructura científica.

\section{¿Por qué la transferencia científica?}

¿En qué consiste esa infraestructura de las ciencias a la cual me refiero y por qué? Primero y básicamente, necesitamos la alfabetización científica y la enseñanza de la ciencia a todos los niveles (por lo menos), para ingenieros y tecnólogos. Para esto se necesitan maestros inspirados, y nadie puede ser un maestro inspirado en ciencias, a menos que haya experimentado y creado, por lo menos algo de la ciencia viva, durante cierta parte de su carrera. Para esto se necesitan laboratorios de enseñanza bien equipados y (en esta era presente de una ciencia a altas velocidades), los más modernos libros y revistas. Esta es la mínima infraestructura que todo país necesita.

Luego, deben venir las demandas a las propias comunidades científicas -comunidades nacionales- de los países subdesarrollados, de las agencias de sus gobiernos y de sus pacientes industrias, para asesorías discriminadas sobre qué tecnologías serían relevantes y necesarias para adquirir.

Subsecuentemente, para una minoría de países subdesarrollados, existe la necesidad de científicos en áreas básicas para ayudar a sus colegas de ciencia aplicada en su trabajo. Para cualquier sociedad, los problemas de su agricultura, o sus plagas y enfermedades, o su materia prima local, debe ser resuelto localmente. Se necesita un énfasis en una base de ciencias básicas de primera clase, para llevar a cabo investigaciones aplicadas en estas áreas. El trabajo en ciencia aplicada, en un país en desarrollo es mucho más difícil que el trabajo en ciencias básicas, simplemente porque uno no tiene cerca o al otro lado de la línea telefónica, personas que puedan decirle lo que uno necesita saber de lo básico, y qué es relevante para el trabajo aplicado que se realiza. 
Y finalmente, en las etapas avanzadas del desarrollo de un país (asumo a través de todo el artículo que nadie con autoridad en países en desarrollo, está lo más mínimamente interesado en el avance del conocimiento por su valor intrínseco, por lo menos no por nacionales del Tercer Mundo), es la necesidad de investigación científica básica por la riqueza que puede de repente proporcionar para la tecnología.

Resumiendo, la tecnología en condiciones modernas no puede florecer si la ciencia no florece también al mismo tiempo. Esto me fue enfatizado recientemente por un físico turco de la Universidad de Samsun, quien recordaba que el Sultán Selim III introdujo estudios de álgebra, trigonometría, mecánica, balística y metalurgia en Turquía en el año 1799, creando escuelas especiales para estas disciplinas con profesores franceses y suecos. Su propósito fue modernizar su ejército y rivalizar a Europa en la creación de armas. Ya que no hubo un énfasis correspondiente en investigación en estas asignaturas, Turquía no tuvo éxito. A largo plazo, en las condiciones de hoy, la tecnología, sin apoyo de la ciencia, simplemente no florecerá.

\section{La situación de las ciencias en el Tercer Mundo}

Vamos a ver la situación de las ciencias y la investigación científica en el Tercer Mundo, exceptuando algunos países (Argentina, Brasil, China e India) que mencioné anteriormente. Puedo ilustrar la situación del ejemplo de mi propio país. En 1951 cuando regresé a Pakistán a enseñar, luego de haber trabajado en Princeton y Cambridge en el campo de la física de alta energía, en un país entonces de 90 millones yo podía conversar con un solo físico que había trabajado en un área parecida. Los números más recientes de la revista Physical Reviews eran anteriores a la II Guerra Mundial. No existían estipendios para ir a simposia o conferencias; la única vez que asistí a una conferencia en el Reino Unido, yo gasté un año de ahorros personales.

Luego de 30 años la situación en Pakistán ha mejorado. Para una población de alrededor de 80 millones hoy día, hay unos 46 físicos investigadores, experimentales y teóricos en las 19 universidades de Pakistán. (Bajo las normas de Estados Unidos el número para una población así sería 100 veces mayor, por ejemplo, unos cinco mil científicos). Estos físicos todavía enfrentan los mismos problemas con respecto a revistas, dinero para publicar y viajes a conferencias; Pakistán aún no es miembro de la Unión Internacional de Física Pura y Aplicada, ya que nuestros administradores cientílicos no creen que podemos gastar US\$1,500.00 en anualidades; a nuestros físicos se les sigue diciendo que toda la ciencia básica -aún las áreas necesarias para física "aplicada"- son un lujo asustante para un país pobre. Sin embargo, comparados con Pakistán -y un grupo privilegiado de unos 30 países- la situación en los otros 60 y pico de otros países subdesarrollados sigue tan trágica como Pakistán en 1951. Lo primero es un problema de números, de un tamaño crítico. El número total de físicos entrenados en investigaciones en muchos de estos países pueden ser contados 
con los dedos de una mano; la elección de subdisciplinas en las cuales pueden haber recibido entrenamiento ha estado condicionada más por la suerte que por diseño. Ellos no constituyen una comunidad.

La creación en Trieste, Italia, del Centro Internacional de Física teórica en 1960 se produjo cuando algunos de nosotros, originarios de países subdesarrollados exigimos a las agencias de las Naciones Unidas, y en particular a la Agencia Internacional de Energía Atómica (IAEA) y a la UNESCO, su asistencia para paliar la situación de la investigación en física teórica. Nos encontramos con incompresiones aún de países desarrollados donde la física avanza. Un delegado de un país desarrollado ante la IAEA llegó tan lejos que dijo: "la física teórica es el Rolls Royce de las ciencias y los países en desarrollo sólo necesitan carretas de bueyes". Para él una comunidad de tan pocos como 50 físicos, entrenados a alto nivel en un país como Pakistán con una población de cerca de 120 millones, eran simplemente 50 personas desperdiciadas. Aun, y dejando de lado lo relacionado con la investigación, el hecho de que estas personas eran los responsables de todas las normas y estandares en el campo completo de la educación en física y matemática se veía como irrelevante. El mismo era un economista, que había logrado colarse en una organización científica como la IAEA. El podía entender que necesitábamos un mayor número de economistas de alto nivel, pero eso de matemáticos y físicos de calidad, era un desperdicio lujoso.

En 1964, cuatro años después que la propuesta fue rechazada y luego de un intenso cabildeo, el IAEA estuvo de acuerdo en la creación de un instituto de física. Sin embargo, la suma asignada fue de US\$55,000 para crear un centro internacional de investigaciones. Afortunadamente el gobierno de Italia produjo un generoso estipendio anual de un tercio de millón de dólares y se decidió establecerlo en Trieste. La comunidad internacional de física nos apoyó todo el tiempo. En la primera reunión del Consejo Científico estuvieron presentes J. R. Oppenheimer, Aage Bohr, Víctor Weisskopt y Sandovalarta. Oppenheimer escribió los estatutos del centro.

El centro inició sus operaciones en 1964. La UNESCO se asoció como igual con el IAEA en 1979. El centro ha florecido desde entonces inclusive con el apoyo de aquellos que dudaron de él en sus inicios.

La mayoría de sus fondos, que ahora llegan a US\$4.5 millones llegan principalmente de Italia, IAEA y la UNESCO. En los 20 años de existencia ha variado sus énfasis de física pura hacia disciplinas básicas en la interfaz de física pura y aplicada. Disciplinas como física de materiales y microprocesadores, física de la energía, física de la fusión, física de reactores, física de energía solar y otras formas no-convencionales de energía, geofísica, biofísica, neurofísica, física del laser, física de océanos y desiertos y análisis de sistemas. Todo esto en adición a los ya clásicos física de alta energía, gravedad-cuántica, cosmología, física nuclear y atómica y matemática. Tales cambios hacia la interfaz entre la 
física pura y la básica aplicada se realizó simplemente porque no existía y no existe ningún otro instituto internacional que responda al hambre científica de los físicos del mundo subdesarrollado.

Durante el 1983, 2,400 físicos vinieron a trabajar al centro de Trieste. 1,400 de ellos procedentes de 90 países subdesarrollados. Aquellos provenientes del subdesarrollo permanecieron como promedio unos dos meses o más en el centro, participando en sus talleres sobre investigación y extensiones de grupos de investigación. Desde el 1981 hemos llevado cuatro de estas extensiones de investigación a países en subdesarrollo: Ghana, Bangladesh, Colombia y ahora Sri Lanka; estos fueron cursos de trabajo y estudio de 4-8 semanas de duración y versaron sobre física del estado sólido, dinámica de monzones, física solar y microprocesadores. Hemos sido pioneros de un tipo de acercamiento que garantiza que los principales físicos de los países subdesarrollados puedan venir al centro, en la época que ellos elijan, por un período que va desde seis semanas hasta tres meses, tres veces en seis años, para trabajar en el ambiente estimulante de sus pares, para cargar sus baterías y retornar a su enseñanza e investigación. No pagamos salarios, sólo el viaje y los gastos. En el presente tenemos 200 asociados de este tipo. Tenemos una red de 102 institutos de física en 41 países en desarrollo en una federación con arreglos de compartir costos. A través de un esquema de Bancos de Libros, hemos distribuido, de donaciones individuales, un total de 2,565 libros y 8,542 copias de revistas recientes a 132 instituciones en 66 países, y hemos iniciado un plan para recoger y distribuir equipos de laboratorios no usados donados por laboratorios de países desarrollados. Con un estipendio del gobierno italiano de un tercio de millón de dólares anuales, hemos colocado 75 físicos experimentales por períodos de 69 meses para investigar en universidades italianas y laboratorios industriales desde 1982. De una manera humilde, el centro ha aumentado la física en general y las comunidades de físicos en países en desarrollo en particular.

\section{Estadios y crecimiento de las Ciencias en el Tercer Mundo}

Basándonos en la experiencia obtenida en la física, podemos dividir los países en desarrollo (excepto Argentina, Brasil, China e India), en tres categorías.

La primera categoría constará de 9 países: Bangladesh, Korea, Malasia, Pakistán, Singapur, Turquía, Egipto, México y Venezuela. Estos países presentan una población de físicas llegando casi a formar masa crítica, así como algunos centros de alta calidad en física donde grupos de científicos pueden realizar investigación independiente. Estos centros están en capacidad de otorgar el nivel de Ph.D. en física en sus propios países.

En la segunda categoría, hay unos 19 países entre los cuales están Irán, Iraq, Jordania, Libano, Indonesia, Filipinas, Sri Lanka, Tailandia, Vietnam, Argelia, Ghana, Kenya, Marruecos, Nigeria, Sudán, Tanzania, Chile, Colombia y Perú. 
Estos países tienen una población modesta de físicos aunque en una universidad dada el número de ellos trabajando es pequeño. No hay grupos de investigaciones como tales, aunque en algunos casos aparecen individuos muy activos. Por lo general el nivel de Ph.D. no se otorga en estos países.

Los restantes 60 países están por debajo "de la línea de pobreza". Con ninguna investigación organizada en física. Algunos con individuos excepcionalmente brillantes, a quienes elegimos como asociados del Centro de Trieste para el día cuando la investigación activa se inicie en sus países. Quiero reafirmar una vez más, que éstas son impresiones basadas en nuestra experiencia en Trieste. Ningún otro significado debe leerse en estas líneas.

\section{Modalidades para el Crecimiento de las ciencias}

Al fin y al cabo, el crecimiento de la ciencia en nuestros paíeses es nuestro problema. Pero en este artículo quiero hablar sólo de la ayuda que podemos esperar de la comunidad científica y de otros en países desarrollados, para avanzar la práctica de la ciencia a alto nivel en el Tercer Mundo. No hay duda que la ayuda externa -particularmente si está organizada- puede ser la diferencia crucial.

Los laboratorios de investigación y los departamentos de universidades de países desarrollados podrían también ayudar desarrollando vínculos federativos con sus colegas y financiando la organización de visitas de sus miembros a las instituciones de países en desarrollo. Pudieran crear las relaciones que hemos desarrollado nosotros aquí en Trieste (donde un físico de alto nivel que labora en un país en desarrollo pasa a ser miembro de nuestra infraestructura de recursos humanos al tener el derecho de venir tres veces en seis años) por lo menos para sus exalumnos.

Que se me perdone por pensar de la siguiente manera: que las instituciones de física de países desarrollados consideren contribuir a su manera (en concreto) de acuerdo a las normas de la bien conocida fórmula de las Naciones Unidas, donde los países avanzados se comprometen a gastar el $0.7 \%$ de PIB para el desarrollo mundial. En fin de cuentas, es un dilema moral el que los segmentos más desarrollados de la comunidad científica deban estar dispuestos a ayudar a sus propios colegas, no sólo materialmente para que permanezcan siendo buenos científicos, sino también uniéndonos a ellos en la batalla dentro de sus propios países para obtener reconocimiento como profesionales válidos, que son importantes para el desarrollo de sus países y del mundo.

Permítaseme considerar ahora el problema de la ayuda a largo plazo que las agencias de las Naciones Unidas puedan ofrecer respecto a la construcción de infraestructura científica, en áreas de su competencia. Particularmente deseo enfatizar el papel de la modalidad con la que personalmente estoy más familiarizado, los centros internacionales de investigaciones. No hay ninguna 
duda de que el mundo subdesarrollado necesita hoy día de instituciones internacionales de investigaciones como por ejemplo en el área aplicada el Instituto de Investigación sobre trigo y arroz; y en el lado científico, centros como el Centro Internacional de Ecología y Fisiología de Insectos (ICIPE) en Nairobi. Sin internacionalización la ciencia no puede florecer, no se pueden garantizar los estándares, el mantenimiento al día con las nuevas ideas, la transferencia continua de la ciencia por las personas que la crean y que van a dichos centros movidos por su idealismo.

Recientemente se han fundado un Centro Internacional de Matemática en Niza, un Centro Internacional de Ciencias en Sri Lanka, uno en Turquía y otro en Venezuela. Un Centro Internacional de Física dirigido hacia América Latina fue formalmente inaugurado en Colombia por el presidente de dicha República hace unos meses. La Organización de Desarrollo Industrial de las Naciones Unidas (UNIDO) está en vías de crear dos centros internacionales en el campo de la biotecnología, uno localizado en Trieste y uno en India.

El director ejecutivo de UNIDO, Dr. Abd-El Rahman Khane, de Argelia, quien en una visita a Trieste, fue impresionado con nuestro ejemplo de interacción de física pura y aplicada, concibió la idea de que las condiciones estaban maduras para crear uno o más centros similares para biotecnología en el Tercer Mundo. UNIDO estableció un concurso que produjo ofertas de parte de Italia, España, India, Pakistán, Egipto, Tailandia y Cuba.

Tres reuniones interministeriales se celebraron para elegir el país; el mes pasado, Delhi y Trieste fueron finalmente seleccionados para la localización de dos centros.

Para mí una de las principales características de la situación de las bio-ciencias es el hecho de que muchos de los países del Tercer Mundo fueron capaces de presentar ofertas viables con sus propios recursos. Personalmente, siento la decisión de los ministros reunidos, quienes por un voto mayoritorio se opusieron a que los perdedores (Egipto, Tailandia, Pakistán y España) pudiesen adquirir el status de centros asociados. Estos países querían desesperadamente aunque fuese un status de subsidiario para poder percibir los beneficios de la internacionalización de sus esfuerzos locales. Yo espero que prontamente esto sea corregido y que éstas y otras ofertas sean aceptadas. El punto que quiero aclarar es que aun países con tradición moderada de investigación científica están comenzando a mostrar interés en recibir y dirigir centros científicos de las Naciones Unidas.

En este contexto, para mostrar el papel que agencias como el Banco Mundial o el Fondo Monetario Internacional pueden jugar, consideramos la siguiente cita de una reciente biografía del Banco Mundial de E.S. Mason y R.E. Asher: "La UNESCO ha venido ofreciendo asesorías valiosas sobre la planificación educativa por años, antes de que el Banco Mundial entrase en 
dicho campo... A veces, algunas partes de estas asesorías eran aceptadas, pero se produjo un notable aumento en la atención dada a la planificación educativa cuando se aclaró que algunos proyectos tenían cierta oportunidad de ser financiados por el Banco Mundial".

Además de la planificación educativa, además de la ayuda para el desarrollo de la agricultura científica, desearía que el Banco Mundial enfatizara a los países en desarrollo que la vía más rápida hacia la prosperidad financiera hoy día radica en áreas científicas (alta tecnología), por ejemplo la microelectrónica, programación de computadoras y por el estilo y la mayor inversión necesaria en estas áreas es la creación de recursos humanos con alta cultura científica. El día que los empresarios (gobiernos o sector privado de países de economías subdesarrolladas) comiencen a entender esto, la salvación de nuestras economías se iniciará.

Para resumir, mi sensación es que casi todos los países en desarrollo tienen un problema científico y tecnológico que necesita de científicos expertos para su solución. Creo muy firmemente que el sistema de las Naciones Unidas debe tomar la iniciativa con este legítimo movimiento hacia la internacionalización de la ciencia dentro del mundo subdesarrollado y para el mundo subdesarrollado.

Finalmente, veamos el papel de las agencias de ayuda. Mi ruego a éstas en todas partes sería que asumieran una actitud a largo plazo con respecto al desarrollo de la ciencia. Ellas representan mucha influencia que deben usar para asegurar que se construya una adecuada infraestructura en los países que ayuden, y que a las comunidades científicas de esos países les sea permitido jugar el papel que les corresponde en el proceso del desarrollo.

En este contexto, se puede señalar que una de las modalidades para la transferencia científica es la creación de una Fundación Internacional para la Ciencia que ofrezca estipendios a científicos individuales en los países en desarrollo. Una Fundación Internacional de Ciencia con estos propósitos fue creada por la sugerencia de Roger Revelle, Pierre Auger, Robert Marshak y yo en 1972 en Estocolmo.

Esta fundación es actualmente apoyada por Suecia, Canada, E.U.A., la República Federal Alemana, Francia, Austria, Holanda, Bélgica, Nigeria, Noruega y Suiza. Sus fondos son ofrecidos para la investigación en las áreas de acuacultura, producción animal, tecnología rural y productos naturales, a científicos individuales de países en desarrollo, en estipendios no mayores de 10,000 dólares cada uno. Desafortunadamente, los fondos totales a disposición son sólo dos millones de dólares. Una fundación similar es realizada por la Organización BOSTID de los E.U.A. la cual es apoyada por las organizaciones del AID. Estas iniciativas no cubren otras ciencias naturales así como tampoco ofrecen fondos para la construcción de infraestructura científica. 
Pensando en las recomendaciones sobre transferencia tecnológica de la Comisión Brand, en 1981 tomé la iniciativa de escribir la siguiente carta a los Jefes de Estado que participaban en la Cumbre de Cancún: "Comprendo que la transferencia tecnológica, con énfasis en problemas energéticos, será uno de los tópicos a discutir en el futuro encuentro entre Jefes de Estado Norte-Sur que se realizará en México. Tristemente, la mayoría de los países en desarrolo necesitan ayuda en la construcción de infraestructura científica a todos los niveles y la Transferencia Científica tiene que acompañar a la transferencia tecnológica si esta última quiere sacar raíces en nuestros países. La comunidad científica en el norte puede, y estoy seguro que lo hará, ayudar en la construcción de la correspondiente comunidad del sur, si es que es movilizada para hacerlo".

Sugeri la creación de una fundación científica Norte-Sur para construir un movimiento dirigido a la transferencia científica, con fondos a su disposición de un orden de por lo menos similar a la Fundación Ford (entre 100 y 200 millones al año). Esta fundación debe ser dirigida por la comunidad científica mundial para la investigación y el entrenamiento en ciencias básicas en países en desarrollo.

Recibí respuestas educadas de apoyo a mi petición de todos los jefes de Estado de los países desarrollados; de los países subdesarrollados sólo la Sra. Gandhi respondió (esto quizás enfatiza una vez más la marginalidad de la empresa científica en el Tercer Mundo).

Déjenme decir, sin embargo, que vale bien la pena construir tal modalidad, particularmente desde que la Fundación Ford, en una carta al Secretario de la recientemente fundada Academia de Ciencias del Tercer Mundo nos ha comunicado que la investigación científica en países en desarrollo no constituye ya un área prioritaria para la propia Fundación Ford.

En las ciencias, como en otras esferas, este mundo nuestro está dividido entre ricos y pobres. La mitad rica (el norte industrializado y los países de economía centralizada, con una producción de US\$5 trillones) gastan un $2 \%$, aproximadamente US $\$ 100$ billones en ciencia no militar e investigación para el desarrollo. La restante mitad de la humanidad (el Sur pobre con una quinta parte de esta producción, alrededor de un trillón de dólares) no gasta más de 2,000 millones de dólares ( 2 billones) en ciencia y tecnología. Atendiendo al \% de los países ricos, los pobres deberían gastar diez veces más, unos 20 billones. En la conferencia sobre ciencia y tecnología auspiciada por la ONU en Viena en 1979, los países más pobres solicitaron ayuda para aumentar sus gastos anuales de 2 a 4 billones. Obtuvieron promesas, no de 2 billones o de un billón, sino sólo de la séptima parte. Como todos sabemos aún estas promesas no han sido cumplidas y el Sistema de Fondos de la ONU para Ciencia y Tecnología para el Desarrollo no tiene fondos adecuados. Contrástese esto con el gasto militar. Cada submarino nuclear cuesta 2 billones de dólares y existen por lo 
menos 100 en los océanos mundiales. Unos quinientos centros como el mío en Trieste podrían ser subvencionados por un año con el costo de un solo submarino.

Permítaseme terminar citando al gran místico del siglo 17 John Donne, un hombre que creía en el estado moral del hombre y el ideal internacionalista. "Ningún hombre es una isla, completamente (dueño) de sí mismo; cada hombre es un pedazo de un continente, una parte del todo; si la mar borra un terruño, Europa llega a menos, como si fuera un promontorio, como si fuera territorio tuyo o tus amigos; la muerte de cualquier hombre me disminuye porque estoy comprometido con la humanidad y, por consiguiente, nunca mandes a preguntar para saber por quién dobla la campana: dobla por ti". 